\title{
ESTUDO FITOQUÍMICO DAS CASCAS DAS RAÍZES DE Zanthoxylum rigidum Humb. \& Bonpl. ex Willd (RUTACEAE)
}

Sally Katiuce Moccelini, Virgínia Claudia da Silva, Eliane Augusto Ndiaye e Paulo Teixeira de Sousa Jr.*

Departamento de Química, Universidade Federal de Mato Grosso, 78060-900 Cuiabá - MT, Brasil

Paulo Cezar Vieira

Departamento de Química, Universidade Federal de São Carlos, CP 676, 13560-970 São Carlos - SP, Brasil

Recebido em 27/2/08; aceito em 7/8/08; publicado na web em 20/1/09

\begin{abstract}
PHYTOCHEMICAL STUDY FROM ROOT BARKS OF Zanthoxylum rigidum Humb. \& Bonpl. ex Willd (RUTACEAE). Chemical investigation from root barks of Z. rigidum, resulted in the isolation of lupeol, a mixture of steroids campesterol, sitosterol, stigmasterol, sacarose, hesperidin, $N$-methylatanine and 6-acetonyldihydrochelerythrine. Their structures were established by spectral data analysis. No previous work has been reported on Z. rigidum species.
\end{abstract}

Keywords: Zanthoxylum rigidum; alkaloids; flavonoid.

\section{INTRODUÇÃO}

O gênero Zanthoxylum (Rutaceae) compreende mais de 200 espécies e encontra-se distribuído em todo mundo. ${ }^{1}$ Quimicamente, este gênero é caracterizado pela presença de alcalóides, ${ }^{2-7}$ cumarinas, ${ }^{5,6,8}$ lignanas, ${ }^{4,9}$ amidas ${ }^{10,11}$ e terpenos. ${ }^{5,6,12}$ Sob o ponto de vista farmacológico, ao gênero Zanthoxylum são atribuídas diversas atividades, tais como, antichagásica ${ }^{2}$ tripanocida ${ }^{9}$ antiplasmódica, ${ }^{7}$ anti-HIV ${ }^{12}$ antiinflamatória, ${ }^{8}$ anti-helmíntica, ${ }^{11}$ entre outras de grande interesse medicinal. A espécie Zanthoxylum rigidum Humb. \& Bonpl. ex Willd. é conhecida popularmente na região amazônica como hualaja e sua madeira é utilizada na construção de casas. ${ }^{13}$ No Pantanal, espécies de Zanthoxylum em geral, são denominadas de mamica-de-cadela ou mamica-de-porca.

Neste trabalho registra-se o resultado do primeiro estudo fitoquímico das cascas das raízes de $Z$. rigidum, descrevendo o isolamento e a identificação do triterpeno lupeol, dos esteróides campesterol, estigmasterol e sitosterol, da sacarose (1), do flavonóide hesperidina (2) e dos alcalóides $N$-metilatanina (3) e 6-acetonildiidroqueleritrina (4).

\section{RESULTADOS E DISCUSSÃO}

O extrato hexânico das cascas das raízes de Z. rigidum submetido à cromatografia em coluna de sílica gel resultou no isolamento do lupeol, da mistura dos esteróides campesterol, estigmasterol e sitosterol e do alcalóide $N$-metilatanina (3). A mesma técnica cromatográfica aplicada ao extrato metanólico forneceu novamente o lupeol e o alcalóide $N$-metilatanina (3), além do dissacarídeo sacarose (1), o flavonóide hesperidina (2) e o alcalóide 6-acetonildiidroqueleritrina (4).

A estrutura do lupeol foi identificada pela análise dos espectros de RMN de ${ }^{1} \mathrm{H}$ e ${ }^{13} \mathrm{C}$, comparação com padrões usando CCD e com dados da literatura. ${ }^{14}$ Para confirmação da composição, a mistura dos esteróides campesterol, estigmasterol e sitosterol foi submetida à CG-EM, cuja identificação foi realizada através de comparação com amostras autênticas do padrão e análise dos fragmentos de massas obtidos de cada componente da mistura. No espectro de massas de cada uma das substâncias foi possível observar picos correspondentes ao íon molecular em $m / z, 400\left(16 \%, \mathrm{C}_{29} \mathrm{H}_{36} \mathrm{O}\right), 412\left(7 \%, \mathrm{C}_{29} \mathrm{H}_{48} \mathrm{O}\right) \mathrm{e}$

*e-mail: teixeira@ufmt.br $414\left(16 \%, \mathrm{C}_{29} \mathrm{H}_{50} \mathrm{O}\right)$, respectivamente. Outros picos com fragmentos em $\mathrm{m} / \mathrm{z}, 396,382,367,256,107$ e 55, característicos de esteróides, foram detectados através da comparação do padrão de fragmentação com dados da literatura. ${ }^{15}$

A identificação da sacarose (1), isolada do extrato metanólico, foi realizada com base nos dados de RMN de ${ }^{1} \mathrm{H}$ e ${ }^{13} \mathrm{C}$ (BBD e PENDANT $^{16}$ ) e comparação de seus dados espectroscópicos com os registrados na literatura. ${ }^{17,18}$ Analisando o espectro de $\mathrm{RMN}$ de ${ }^{1} \mathrm{H}$, verificou-se sinais na região de açúcar entre $\delta_{\mathrm{H}} 3,2$ e 4,0, além do sinal atribuído ao hidrogênio ligado ao carbono anomérico em $\delta_{\mathrm{H}} 5,2(d$, $J 3,8 \mathrm{~Hz}$ ). No experimento PENDANT (50 MHz), registraram-se 12 sinais de carbono, sendo três sinais atribuídos aos carbonos metilêni$\cos \delta_{\mathrm{CH} 2} 61,5$ (C-6), 60,1 (C-1') e 62,5 (C-6') e um quaternário em $\delta_{\mathrm{C}}$ 103,7 (C-2'), que confirmaram a estrutura do dissacarídeo sacarose ou $\alpha$-D-glicopiranosil- $\beta$-D-frutofuranosídeo.

A estrutura da flavanona 2 foi definida com base nos dados fornecidos pelos espectros de RMN de ${ }^{1} \mathrm{H},{ }^{13} \mathrm{C}$ (BBD e PENDANT), massas, bem como através da comparação dos valores dos seus deslocamentos químicos com os descritos na literatura. ${ }^{19} \mathrm{O}$ espectro de RMN de ${ }^{1} \mathrm{H}$ mostrou sinais que absorvem na região de hidrogênios aromáticos, além de uma absorção em $\delta_{\mathrm{H}} 12,06$ indicando hidroxila quelada e outra em $\delta_{\mathrm{H}} 3,77$ com integração para 3 hidrogênios, indicando a presença de grupamento metoxílico. Os sinais correspondentes ao par de singletos largos em $\delta_{\mathrm{H}} 6,15(\mathrm{H}-6)$ e $\delta_{\mathrm{H}} 6,12(\mathrm{H}-$ 8 ), formando um sistema $\mathrm{AB}$ no anel $\mathrm{A}$, e um multipleto centrado registrado em $\delta_{H}$ 6,92 atribuído aos hidrogênios H-2', H-5' e H-6', representando um sistema do tipo AMX no anel $\mathrm{B}$, foram verificados na região de hidrogênios aromáticos. Ainda pelo espectro de RMN de ${ }^{1} \mathrm{H}$ foram observados sinais atribuídos a um sistema $\mathrm{ABX}$ na região de hidrogênios alifáticos em $\delta_{\mathrm{H}} 5,49(d d, J 3,11$ e $12,18 \mathrm{~Hz}$, $\mathrm{H}-2), \delta_{\mathrm{H}} 2,77\left(d d, J 3,11\right.$ e $15,11 \mathrm{~Hz}, \mathrm{H}-3$ eq) e $\delta_{\mathrm{H}} 3,07$ ( $d d, J 13,8$ e $12,0 \mathrm{~Hz}, \mathrm{H}-3 \mathrm{ax}$ ) os quais caracterizaram o esqueleto da flavanona. A presença de sinais na região de açúcar $\delta_{\mathrm{H}} 3,09-3,86$, o dubleto em $\delta_{\mathrm{H}}$ $4,52(\mathrm{~J} 7,38 \mathrm{~Hz})$ e o singleto em $\delta_{\mathrm{H}} 4,97$ atribuídos aos hidrogênios anoméricos $\mathrm{H}-1$ " e H-1"', respectivamente, e principalmente o sinal de dubleto em $\delta_{\mathrm{H}} 1,08$ ( $d, J$ 6,0 Hz, H-6") confirmam a presença de uma unidade ramnose na molécula. A análise do espectro de RMN de ${ }^{13} \mathrm{C}$ (PENDANT) confirmou os respectivos carbonos ligados aos hidrogênios representados por esses sinais e os carbonos metínico em $\delta_{\mathrm{CH}} 78,4$ e metilênico em $\delta_{\mathrm{CH} 2} 42,1$, atribuídos aos respectivos 

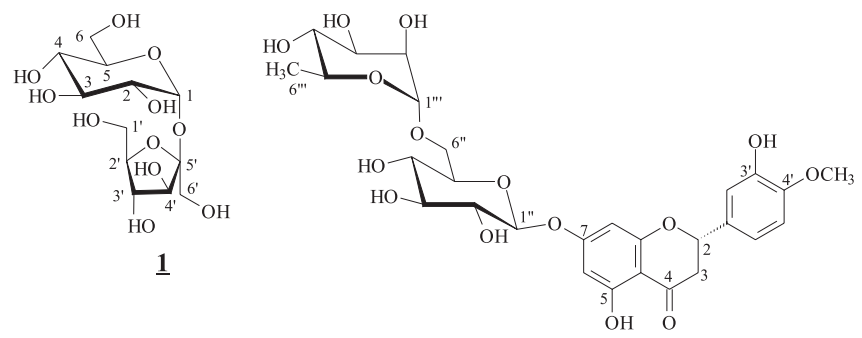

$\underline{2}$

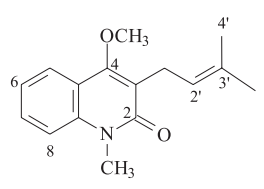

$\underline{\mathbf{3}}$

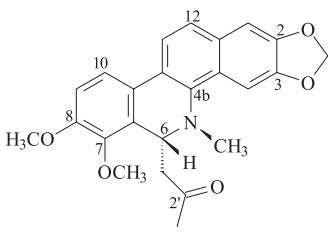

$\underline{4}$ carbonos C-2/C-3 (anel A), os carbonos metínicos não oxigenados-sp ${ }^{2}$ em $\delta_{\mathrm{CH}} 96,4$ e 95,6 atribuídos aos carbonos C-6/C-8 (anel B) e em $\delta_{\mathrm{CH}}$ $114,2,112,0$ e 117,9 relativos aos carbonos C-2'/C-5'/C-6' (anel C). A atribuição de todos esses valores foi compatível ao esqueleto de uma flavanona 7-O-glicosil substituída. O espectro de RMN de ${ }^{13} \mathrm{C}$ também auxiliou na confirmação dos sinais dos carbonos quaternários da unidade aglicona proposta para $2\left[\delta_{\mathrm{C}} 197,0(\mathrm{C}=\mathrm{O}), 163,1(\mathrm{C}-5)\right.$, 165,2 (C-7), 162,5 (C-9), 103,3 (C-10), 130,9 (C-1'), 146,5 (C-3') 147,9 (C-4')]. Outros sinais foram atribuídos à hidroxila fenólica em $\delta_{\mathrm{H}} 12,02(s, \mathrm{HO}-5)$ e 9,11 (s, HO-3'), à metoxila em $\delta_{\mathrm{H}} 3,77(s$, $\left.\mathrm{H}_{3} \mathrm{CO}-4^{\prime}\right)$ e às duas unidades de açúcar em $\delta_{\mathrm{H}} 3,14-3,86,4,97(d, J$ 7,38 Hz, H-1"), 4,52 ( $s, \mathrm{H}-1$ "') e 1,08 (d, $J$ 6,0 Hz, H-6"'). Esses valores de deslocamentos químicos aliados à exibição do pico íon pseudo-molecular $[\mathrm{M}-\mathrm{H}]^{+}$em $\mathrm{m} / z, 609,3$ no espectro de massas (ESI-MS) foram condizentes com a fórmula molecular $\mathrm{C}_{28} \mathrm{H}_{34} \mathrm{O}_{15}$ e confirmaram a estrutura da 7-O- $\beta$-D-rutinosil-3',5'-diidroxi-4'metoxiflavanona ou hesperidina (2).

As estruturas dos alcalóides $\mathbf{3}$ e $\mathbf{4}$ foram definidas com base na análise dos espectros de $\mathrm{RMN}$ de ${ }^{1} \mathrm{H}$ e de ${ }^{13} \mathrm{C}$ uni e bidimensionais e por comparação com os valores descritos na literatura para os alcalóides $N$-metilatanina ${ }^{20}$ e 6 -acetonildiidroqueleritrina, ${ }^{21}$ respectivamente. $\mathrm{O}$ espectro de $\mathrm{RMN}$ de ${ }^{1} \mathrm{H}$ de $\mathbf{3}$ indicou a presença de quatro hidrogênios em sistema aromático, cujos respectivos sinais apareceram em $\delta_{\mathrm{H}} 7,78(d d, J 1,5$ e 7,9 Hz, H-5), 7,48 (ddd, $J 1,5$, $7,1$ e $8,5 \mathrm{~Hz}, \mathrm{H}-7), 7,33$ (d, J 7,2 Hz, H-8) e 7,19 ( $t, J$ 7,1 Hz, H-6). A multiplicidade dos sinais em $\delta_{\mathrm{H}} 5,28\left(t, \mathrm{H}-2^{\prime}\right)$ e $3,40\left(d, \mathrm{H}-4^{\prime}\right)$ com valor da constante de acoplamento $\mathrm{J}=6,8 \mathrm{~Hz}$ foi compatível com a relação entre os hidrogênios ligados aos carbonos C-1' e C-2'. Além desses sinais, observou-se a presença dos singletos em $\delta_{H} 1,69,1,82$ e 3,67, atribuídos aos grupamentos metila, sendo o último valor correspondente ao grupamento $N$-metil e, em $\delta_{\mathrm{H}} 3,67$, equivalente à metoxila. No espectro de $\mathrm{RMN}$ de ${ }^{13} \mathrm{C}\left(\mathrm{BBD}\right.$ e DEPT $\left.135^{\circ}\right)$ entre outros sinais, observaram-se os sinais dos carbonos metilênico em $\delta_{\mathrm{CH}_{2}} 23,9\left(\mathrm{C}-1^{\prime}\right)$, metílicos $\delta_{\mathrm{CH}_{3}} 25,3$ (C-5'), 17,6 (C-4'), 29,2 ( $\left.\mathrm{N}-\underline{\mathrm{CH}}_{3}\right)$ e $61,3\left(\mathrm{OCH}_{3}\right)$, olefínico $\delta_{\mathrm{CH}} 121,24\left(\mathrm{C}-2\right.$ '), metínicos $\delta_{\mathrm{CH}} 121,2\left(\mathrm{C}-2^{\prime}\right)$, 122,8 (C-5), 121,4 (C-6), 129,6 (C-7) e 113,6 (C-8) e quaternários 131,9 (C-3'), 117,9 (C-3), 159,7 (C-4), 122,0 (C-4a), 138,5 (C-8a) e $163,4(\mathrm{C}=\mathrm{O})$.

Os alcalóides do tipo benzofenantridínicos, semelhantes à 6-acetonildiidro queleritrina (4), são muito comuns no gênero Zanthoxylum. ${ }^{22}$ Os espectros de RMN de ${ }^{1} \mathrm{H}$ da substância 4, incluindo experimento ${ }^{1} \mathrm{Hx}{ }^{1} \mathrm{H}$-COSY, apresentaram multiplicidades e valores das constantes de acoplamento da correlação entre os sinais em $\delta_{\mathrm{H}} 2,25(d d$,

$J=3,60$ e 14,90 Hz, H-1'a), 2,57 (dd, $J=11,20$ e 14,90 Hz, H-1'b) e 5,04 ( $d d, J=3,60$ e $11,2 \mathrm{~Hz}, \mathrm{H}-6)$; entre $\delta_{\mathrm{H}} 6,95(d, J=8,5 \mathrm{~Hz}, \mathrm{H}-9)$ e $\delta_{\mathrm{H}} 7,54(d, J=8,5 \mathrm{~Hz}, \mathrm{H}-10)$ e entre $\delta_{\mathrm{H}} 7,71(d, J=8,5 \mathrm{~Hz}, \mathrm{H}-11)$ e $\delta_{\mathrm{H}} 7,48(d, J=8,5 \mathrm{~Hz}, \mathrm{H}-12)$. Outros sinais de destaque no espectro de RMN de ${ }^{1} \mathrm{H}$ foram os sinais do grupamento $N$-metil em $\delta_{\mathrm{H}} 2,64$, metoxi em $\delta_{\mathrm{H}} 3,92,3,95\left(\mathrm{OCH}_{3}\right)$, metilenodioxi em $\delta_{\mathrm{H}} 6,04(s)$, além dos singletos em $\delta_{\mathrm{H}}$ 7,10 e 7,51 atribuídos aos hidrogênios H-4 e H-1, respectivamente. A análise do espectro de HSQC permitiu atribuir inequivocamente todos os valores de deslocamento químico dos carbonos hidrogenados (ver Parte Experimental). O espectro de RMN de ${ }^{13} \mathrm{C}$ aliado ao espectro HMBC confirmou a localização das metoxilas pela correlação ${ }^{3} \mathrm{~J}$ entre o sinal em $\delta_{\mathrm{H}} 3,92$ com C-7 $(152,1)$ e $\delta_{\mathrm{H}} 3,95$ com C-8 $(145,5)$, da acetonila ${ }^{3} \mathrm{~J}$ entre H-6 $(5,04)$ com C=O $(207,5)$ e metilenodioxi ${ }^{3} \mathrm{~J}$ entre $\mathrm{O}_{2} \mathrm{CH}_{2}(6,04)$ com C-2 $(147,5)$ e C-3 $(148,1)$. A análise por ES-MS sugeriu a fórmula molecular $\mathrm{C}_{24} \mathrm{H}_{23} \mathrm{NO}_{5}$, devido à presença do íon pseudo-molecular $[\mathrm{M}+\mathrm{H}]^{+} \mathrm{em} \mathrm{m} / z$ 406,6, indicando também a presença de número impar de nitrogênios e confirmou a proposta para a estrutura da 6-acetonildiidroqueleritrina (4).

\section{PARTE EXPERIMENTAL}

\section{Procedimentos experimentais}

Os pontos de fusão foram determinados em medidor digital Mettler FP 80-Hot Stage acoplado em microscópio binocular Olympus. Os espectros de massas de baixa resolução foram efetuados em cromatógrafo em fase gasosa acoplado à espectrometria de massas (CG-EM), modelo QP 5000 da Shimadzu, utilizando sistema de ion trap e ionização por impacto de elétrons a $70 \mathrm{eV}$ e espectros de massas de alta resolução por ionização eletrospray foram efetuados em espectrômetro Quatro LC-Micromass UK (ESI-MS). As análises polarimétricas foram obtidas em polarímetro Perkin Elmer modelo 341 , e os solventes foram clorofórmio, metanol e DMSO grau analítico e água Milli-Q. Os espectros de Ressonância Magnética Nuclear, ${ }^{1} \mathrm{He}{ }^{13} \mathrm{C}$ (incluindo experimentos em 2D) foram registrados em espectrômetros Bruker ARX-400 ( ${ }^{1} \mathrm{H}: 400 \mathrm{MHz}$ e ${ }^{13} \mathrm{C}$ : $\left.100 \mathrm{MHz}\right)$; Bruker AC-200 ( ${ }^{1} \mathrm{H}: 200 \mathrm{MHz}$ e $\left.{ }^{13} \mathrm{C}: 50 \mathrm{MHz}\right)$ e Jeol JNM-GX-400 $\left({ }^{1} \mathrm{H}: 400 \mathrm{MHz}\right.$ e ${ }^{13} \mathrm{C}$ : $100 \mathrm{MHz}$ ), utilizando $\mathrm{CDCl}_{3}, \mathrm{D}_{2} \mathrm{O}, \mathrm{CD}_{3} \mathrm{OD}$ ou DMSO- $\mathrm{d}_{6}$ como solventes e TMS como referência interna. Nas separações cromatográficas em coluna aberta usou-se sílica gel 60 (Merck e Aldrich); nas análises com camada delgada e preparativa utilizou-se sílica gel $60 \mathrm{PF}_{254}$ (Merck e Aldrich) com granulação adequada e revelação sob luz UV (254 e 365 nm), vanilina ácida, reagente de Dragendorff ou exposição em vapores de iodo.

\section{Material vegetal}

As raízes de Zanthoxylum rigidum foram coletadas em março de 2001 na fazenda N. S de Fátima, Poconé-Porto Cercado km 8, município de Poconé (MT). A ratificação taxonômica foi realizada pela Prof ${ }^{\text {a }}$. Dra ${ }^{\text {a }}$. F. Melo do Instituto Nacional de Pesquisas da Amazônia (INPA), sendo que no Herbário Central da UFMT se encontra catalogada uma amostra testemunho (exsicata $n^{\circ} 24.081$ ).

\section{Extração e isolamento}

Após secagem e trituração, 520,0 g das cascas das raízes de $Z$. rigidum foram submetidas à extração através de maceração contínua com hexano e metanol à temperatura ambiente. A retirada do solvente foi feita em evaporador rotativo, originando os extratos hexânico (15,6 g) e metanólico $(68,0 \mathrm{~g})$. O extrato hexânico foi cromatografado em coluna de sílica gel 60 eluída inicialmente com hexano aumentando-se gradativamente a polaridade com AcOEt e $\mathrm{MeOH}$, finalizando com $\mathrm{MeOH}$. Foram obtidas 204 frações de aproximadamente $25 \mathrm{~mL}$ cada, 
reunidas conforme a similaridade em 22 grupos (G1-G22). A fração G4 (1,2 g) foi purificada em CC sílica gel eluída com hexano, misturas de hexano e AcOEt, AcOEt, misturas de AcOEt e $\mathrm{MeOH}$ e $\mathrm{MeOH}$ obtendo-se 24 frações, sendo que das frações 4-7 se obteve um sólido branco correspondente ao lupeol $\left(30,0 \mathrm{mg}\right.$, p.f. $\left.212-214{ }^{\circ} \mathrm{C}\right)$. A fração G5 (150,0 mg) forneceu cristais em forma de agulhas incolores que foram submetidos à análise por CG-EM, obtendo-se assim a mistura dos esteróides campesterol, estigmasterol e sitosterol (5,0 mg). As frações G6-G9 forneceram a substância 2 (1,2 g, p.f. $132,5-134,2{ }^{\circ} \mathrm{C}$, óleo amarelo-esverdeado). O extrato metanólico $(68,0 \mathrm{~g})$ foi cromatografado em coluna de sílica gel utilizando-se os gradientes hexano, misturas de hexano e AcOEt, AcOEt e misturas de AcOEt e $\mathrm{MeOH}$, $\mathrm{MeOH}$ e misturas de $\mathrm{MeOH}$ e dietilamina. Foram obtidas 345 frações que foram analisadas e reunidas por CCD em 35 grupos (F1-F35). A partir do fracionamento por CC da fração F2 $(290,0 \mathrm{mg})$ foi novamente obtido o triterpeno lupeol (10,0 mg). A fração F5 (510,0 mg) foi repurificada por CC em sílica gel utilizando-se como eluentes, hexano, AcOEt e $\mathrm{MeOH}$, com aumento gradativo de polaridade. Foram obtidas 107 frações, reunidas em 17 frações (F5A-F5R). As frações F5I, F5J e F10 (150,0 mg) foram reunidas por similaridade por conterem a mesma substância majoritária. Estas depois de agrupadas foram purificadas por CCDP eluída com $\mathrm{CH}_{2} \mathrm{Cl}_{2}$ e AcOEt (95:5) e deram origem ao alcalóide 4 (18,0 mg, p.f. 192,2-193,3 ${ }^{\circ} \mathrm{C}$ ). Nas frações F25-F-27 houve a precipitação de um sólido cristalino em forma de cubos, que foram separados do sobrenadante por filtração, correspondente à sacarose $(\mathbf{1}$, $330,0 \mathrm{mg}$, p.f. $\left.180-184^{\circ} \mathrm{C}\right)$. O sobrenadante das frações F25-F27 (7,38 g) foi submetido à CC de sílica gel 60 , eluída com misturas de hexano e AcOEt e misturas de AcOEt e $\mathrm{MeOH}$, da qual foram recolhidas 236 frações que, com base em análise CCD, foram reunidas em 14 grupos (F25/27A-F25/F27N). Nas frações F25/F27J houve a formação de um sólido amorfo amarelado correspondente à substância 2 (144,0 mg). Nas frações F28-F29 também houve precipitação da substância 2 (740,0 mg, p.f. $258-259,2{ }^{\circ} \mathrm{C}$ ).

Sacarose (1): cristais transparentes, p.f. $180-184{ }^{\circ} \mathrm{C},[\alpha]_{\mathrm{D}}^{22^{\circ}}+0,534$ ( $\mathrm{H}_{2} \mathrm{O}$; conc. $\left.0,01 \mathrm{~g} / \mathrm{mL}\right), \mathrm{RMN} d{ }^{13} \mathrm{C}\left(50 \mathrm{MHz}, \mathrm{D}_{2} \mathrm{O}\right) \delta_{\mathrm{C}}: 92,2(\mathrm{C}-1)$, 72,5 (C-2), 74,1 (C-3), 71,2 (C-4), 72,7 (C-5), 61,5 (C-6) [glicose], 60,3 (C-1'), 103,7 (C-2'), 76,5 (C-3'), 69,4 (C-4'), 81,5 (C-5'), 62,5 (C-6') [frutose].

Hesperidina (2): sólido amorfo amarelado, p.f. $258,0-259,2{ }^{\circ} \mathrm{C},[\alpha]_{\mathrm{D}}$ ${ }^{22^{\circ}}$-67,98 (DMSO; conc. 0,011 g/mL), RMN ${ }^{1} \mathrm{H}$ (200 MHz, DMSO$\left.\mathrm{d}_{6}\right) \delta_{\mathrm{H}}($ mult.; $J$ em Hz, H): 5,49 (dd, 3,11 e 15,11, H-2), 2,77 (dd, $3,11$ e $15,11, \mathrm{H}-3 \mathrm{eq}), 3,07$ ( $d d, 13,8$ e 12,0, H-3ax), 6,15 ( $s l, \mathrm{H}-6)$, 6,12 ( $s l, \mathrm{H}-8$ ), 6,92 ( $m, \mathrm{H}-2$ ', H-5', H-6'), 4,52 (d, 7,8, H-1'), 4,97 ( $s, \mathrm{H}-1$ "'), 3,09-3,86 (H-2"-H-5"'), 1,08 ( $d$, 6,0, H-6"), 12,06 ( $s$, $5-\mathrm{OH}), 3,77\left(s, 4^{\prime}-\mathrm{OC}_{3}\right)$. RMN ${ }^{13} \mathrm{C}\left(50 \mathrm{MHz}, \mathrm{DMSO}-\mathrm{d}_{6}\right) \delta_{\mathrm{C}}: 78,4$ (C-2), 42,1 (C-3), 197,0 (C-4), 163,1 (C-5), 96,4 (C-6), 165,2 (C-7), 95,6 (C-8), 162,5 (C-9), 103,3 (C-10), 130,9 (C-1'), 114,2 (C-2'), 146,5 (C-3'), 147,9 (C-4'), 112,0 (C-5'), 117,9 (C-6'), 100,6 (C-1'), 73,0 (C-2”), 76,3 (C-3"), 69,6 (C-4”), 75,5 (C-5”), 66,1 (C-6”), 99,5 (C-1"'), 70,3 (C-2"'), 70,7 (C-3"'), 72,1 (C-4"'), 68,3 (C-5"'), 17,8 (C-6"'), 55,7 (4'-O $\left.\underline{\mathrm{CH}}_{3}\right)$.

$N$-metilatanina (3): óleo amarelo esverdeado, p.f. $132,5-134,2{ }^{\circ} \mathrm{C}$, $\mathrm{RMN}{ }^{1} \mathrm{H}\left(200 \mathrm{MHz}, \mathrm{CDCl}_{3}\right) \delta_{\mathrm{H}}$ (mult.; $J$ em Hz, H): 7,78 (dd, 1,5 e 7,1, H-5), 7,19 ( $t, 7,1, \mathrm{H}-6), 7,48$ ( $d d d, 1,5,7,1$ e 8,5, H-7), 7,33 (d, 7,2, H-8), 3,40 ( $d, 6,8, \mathrm{H}^{-1}$ '), 5,28 ( $\left.t, 6,8, \mathrm{H}^{\prime} 2^{\prime}\right), 1,69$ ( $\left.s, \mathrm{H}-4^{\prime}\right), 1,82$ ( $s, \mathrm{H}-5$ '), 3,89 ( $\left.s, 4^{\prime}-\mathrm{OCH}_{3}\right), 3,67\left(s, N-\mathrm{CH}_{3}\right) . \mathrm{RMN}{ }^{13} \mathrm{C}(50 \mathrm{MHz}$, $\left.\mathrm{CDCl}_{3}\right) \delta_{\mathrm{C}}: 163,4(\mathrm{C}-2), 117,2(\mathrm{C}-3), 159,7(\mathrm{C}-4), 122,0(\mathrm{C}-4 \mathrm{a})$, 122,8 (C-5), 121,4 (C-6), 129,6 (C-7), 113,6 (C-8), 138,5 (C-8a), 23,9 (C-1'), 121,2 (C-2'), 131,9 (C-3'), 17,6 (C-4'), 25,3 (C-5'), 61,3 $\left(4^{\prime}-\mathrm{OCH}_{3}\right), 29,2\left(\mathrm{~N}-\underline{\mathrm{CH}}_{3}\right)$. 6-acetonildiidroqueleritrina (4): sólido bege-claro, p.f. 192,2-193,3 ${ }^{\circ} \mathrm{C},[\alpha]_{\mathrm{D}}{ }^{22^{\circ}}-5,560\left(\mathrm{CHCl}_{3}\right.$; conc. 0,014 g/mL), RMN ${ }^{1} \mathrm{H}(400 \mathrm{MHz}$, $\left.\mathrm{CDCl}_{3}\right) \delta_{\mathrm{H}}$ (mult; $J$ em Hz, H): 7,51 ( $\left.s, \mathrm{H}-1\right), 7,10(s, \mathrm{H}-4), 5,04$ (dd, 3,6 e 11,2, H-6), 6,95 (d, 8,5, H-9), 7,54 (d, 8,5, H-10), 7,71 (d, 8,5, H-11), 7,48 (d, 8,6, H-12), 2,57 (dd, 11,2 e 14,9, H-1'a), 2,25 ( $d d, 3,6 \mathrm{e}$ 14,9, H-1'b), 6,04 ( $\left.s, \mathrm{O}_{2} \underline{\mathrm{CH}}_{2}\right), 2,06$ ( $\left.s, \mathrm{COCH}_{3}\right), 3,92\left(s, 7-\mathrm{OCH}_{3}\right), 3,95$ $\left(s, 8-\mathrm{OC} \underline{\mathrm{H}}_{3}\right), 2,64\left(N-\underline{\mathrm{C}}_{3}\right)$. RMN ${ }^{13} \mathrm{C}\left(100 \mathrm{MHz}, \mathrm{CDCl}_{3}\right) \delta_{\mathrm{C}}: 100,6$ (C-1), 147,5 (C-2), 148,1 (C-3), 104,3 (C-4), 127,3 (C-4a), 124,8 (C-4b), 54,9 (C-6), 139,3 (C-6a), 152,1 (C-7), 145,5 (C-8), 111,5 (C9), 118,7 (C-10), 128,2 (C-10a), 123,3 (C-10b), 119,7 (C-11), 123,8 (C-12), 131,0 (C-120), 46,8 (C-1'), 207,5 (C-2'), 31,1 (C-3'), 55,8 $\left(7-\mathrm{OCH}_{3}\right), 60,9\left(8-\mathrm{O}_{\mathbf{C H}}\right), 101,0\left(\mathrm{O}_{2} \underline{\mathrm{C}} \mathrm{H}_{2}\right), 42,8\left(\mathrm{~N}-\underline{\mathrm{C}} \mathrm{H}_{3}\right)$.

\section{AGRADECIMENTOS}

Ao CNPq, FAPEMAT e Centro de Pesquisa do Pantanal (CPP) pelas bolsas concedidas e pelo apoio financeiro para o desenvolvimento dessa pesquisa e ao Prof. Dr. A. G. Ferreira (UFSCar) pelos espectros obtidos a $400 \mathrm{MHz}$.

\section{REFERÊNCIAS}

1. Talapatra, S. K.; Dutta, S. K.; Talapatra, B.; Phytochemistry 1973, 12, 729.

2. Ferreira, M. E.; Nakayama, H.; Arias, A. R.; Schinini, A.; de Bilbao, N. V.; Serna, E.; Lagoutte, D.; Soriano-Agatón, F.; Poupon, E.; Hocquemiller, R.; Fournet, A.; J. Ethnopharmacol. 2007, 109, 258.

3. Hu, J.; Zhang, W. D.; Shen, Y. H.; Zhang, C.; Xu, L.; Liu, R. H.; Wang, B.; Xu, X. K.; Biochem. Syst. Ecol. 2007, 35, 114.

4. Rahman, M. M.; Islam, M. A.; Khondkar, P.; Gray, A. I.; Biochem. Syst. Ecol. 2005, 33, 91 .

5. Arruda, M. S. P.; Fernandes, J. B.; Vieira, P. C.; da Silva, M. F. G. F.; Pirani, J. R.; Biochem. Syst. Ecol. 1992, 20, 173.

6. Oliveira, E. L.; Freitas, P. C.; Guedes, M. L. S.; Velozo, E. S.; Rev. Bras. Farmacogn. 2002, 12, 29.

7. Jullian, V.; Bourdy, G.; Georges, S.; Maurel, S.; Sauvain, M.; J. Ethnopharmacol. 2006, 106, 348.

8. Chen, I. S.; Lin, Y. C.; Tsai, I. L.; Teng, C. M.; Ko, F. N.; Ishikawa, T.; Ishii, H.; Phytochemistry 1995, 39, 1091.

9. Bastos, J. K.; Albuquerque, S.; Silva, M. L. A.; Planta Med. 1999, 65, 541.

10. Chen, I. S.; Chen, T. L.; Lin, W. Y.; Tsai, I. L.; Chen, Y. C.; Phytochemistry 1999, 52, 357.

11. Navarrete, A.; Hong, E.; Planta Med. 1996, 62, 250.

12. Cheng, M. J.; Lee, K. H.; Tsai, I. L.; Chen, I. S.; Bioorg. Med. Chem. 2005, 13, 5915 .

13. Duke, J. A.; Vasques, R.; Amazonian Ethnobotanical Dictionary, CRC Press Inc.: Boca Raton, 1994.

14. Mahato, S. B.; Kundu, A. P.; Phytochemistry 1994, 37, 1517.

15. Branco, A.; Pizzolatti, M. G.; Quim. Nova 2002, 25, 15.

16. Homer, J.; Perry, M. C.; J. Chem. Soc. Chem. Commun. 1994, 373.

17. Agrawal, P. K.; Phytochemistry 1992, 31, 3307.

18. Breitmaier, E.; Voelter, W.; Carbon-13 NMR spectroscopy-High-resolution methods and applications in organic chemistry and biochemistry, $3^{\text {rd }}$ ed., VCH: Weinheim, 1989.

19. Ferreira, D. T.; Álvares, P. S. M.; Houghton, P. J.; Braz-Filho, R.; Quim. Nova 2000, 23, 42.

20. Fauvel, M. T.; Gleye, J.; Moulis, C.; Blasco, F.; Stanislas, E.; Phytochemistry 1981, 20, 2059.

21. Julián, A.; Delgado, G.; Rev. Soc. Quim. Mex. 2001, 45, 189.

22. Waterman, P. G.; Grudon, M. F.; Chemistry and Chemical Taxonomy of the Rutales, Academic Press: London. 